\title{
OPTIMIZATION OF STRUCTURAL ELEMENTS
}

\author{
Jean-Louis ARMAND \\ Institut de Recherches de la Construction Navale \\ 47, rue de Monceau - 75008 PARIS, FRANCE \\ also
}

Ecole Polytechnique, 5 rue Descartes - 75005 PARIS, FRANCE

\section{Abstract}

A numerical method for the solution of structura1 optimization problems involving ordinary differential equations is presented for a simple situation where the constraint is of an aeroelastic nature. The method is adapted from optimal control theory and has proven successful in a number of structural optimization problems. Its extension to two dimensional structures is outlined; 1imitation to situations involving plates, however, is emphasized. It is assumed that the instability exhibited by the optimality condition is related to the fact that plates cannot in general achieve global extrema. Suggestions for further research in this area are presented.

\section{INTRODUCTION}

The general theory of optimization finds a privileged field of application within the framework of Mechanics. Optimization of structural design, in particular, has raised considerable interest in recent years. The available literature concerned with this subject is vast. A comprehensive and organized review up to 1972 can be found in an article by Niordson and Pedersen (1972). Another excellent survey, concerned with constraints of a dynamic nature only, has been provided by Pierson (1972). Since then the flow of new research publications has continued steadily. A new phase seems now to have been reached with the apparition of monographs by Moe and Gisvold (1971), Hemp (1973), Gallagher and $z$ ienkiewicz (1973), Distefano (1974), Majid (1974), among others. Research in structural optimization mainly follows two separate directions. The first approach, sometimes termed structural synthesis, is concerned with the optimization of discrete or discretized structures; it is cast as a mathematical programming problem of extremizing an objective function subject to several equality or inequality constraints. 
For a more complete description, the reader is referred to the papers by Schmit (1971) or Fox (1973), as well as to the texts by Moe and Gisvold (1971) or Gallagher and Zienkiewicz (1973) already mentioned. Although relatively complex structures can be considered, the number of design variables remains, unfortunately, very limited for practical reasons. The second approach is concerned with the optimal design of continuous structural elements, the properties of which are amenable to description by ordinary (one-dimensional structures) or partial (twoand three-dimensional structures) differential equations. A considerable literature exists in this field, although it is systematically ignored by those advocating the mathematical programming approach. This is rather unfortunate, since the continuous approach has the merit of raising basic questions such as the existence, uniqueness and characterization of the solution, questions which cannot be answered by mathematical programming techniques alone. Moreover, the constitution of a library of optimal structural members under a variety of constraints and boundary conditions would provide the practicing designer with efficient ideas in situations where intuition alone is of little help.

The present paper is concerned with the minimum-mass design of elastic structures whose behaviour is described by ordinary differential equations (no mention will be made of so-called plastic design, which is quite a different subject altogether). Despite a large existing literature, very few numerical solutions for structural elements have been presented, and the majority of those are of academic interest only. We present in what follows a numerical solution procedure borrowed from optimal control theory, which has proven quite useful in situations where the conventional variational approach leads to a set of complex nonlinear equations.

\section{OPT IMAL DESIGN OF CONTINUOUS STRUCTURAL ELEMENTS}

Application of the classical variational calculus to structural optimization dates as far back as Lagrange (1770-1773) who investigated the ideal shape that a colum should possess in order to sustain its own weight. In this approach, the resulting equations are unfortunately quite complex and usually nonlinear. It is extremely rare to find a closed-form solution. A relative exception is the class of so-called linear stiffness structures, for which both stiffness and weight are linear in the design variable (cross-sectional area or thickness) ; 
this is the case of sandwich construction in particular. The paper by Prager and Taylor (1968) contains an exhaustive treatment of this subject. A tutorial presentation can be found in Dym (1974). Genera11y, however, the investigator is left with a complex two-point boundary value problem (TPBVP) to solve in the case of one-dimensional elements, or a system of nonlinear partial differential equations in the case of two- or three-dimensional situations.

Application of optimal control theory to structural optimization was independently suggested by Dixon (1967), Ashley and McIntosh (1968) and Haug (1969). It has up to now hardly been realized that the designer has at his disposal the powerful tools which have been developed for the solution of similar problems arising in control theory, described in the book by Bryson and Ho (1969), among others. The idea is to cast the optimization problem in a form amenable to conventional treatment through the introduction of state and design (control) variables. The optimality conditions are then derived through an extension of classical variational methods (a particular case of the more general pontryagins's Maximum Principle). The resulting system of equations is then solved by an iterative procedure.

A systematic presentation of the above points as well as an evaluation of the various numerical schemes will be found in a paper by Armand (1973), who also presents a review of the field. McIntosh (1974) also described the foundations of the method in a review paper in which comparisons with discrete solutions can be found. The approach presented here will be best described through a simple example where the constraint is of an aeroelastic nature, first suggested by Ashley and McIntosh (1968) and treated in detail by Armand and Vitte (1970), in which results of computation can be compared with the exact analytical solution.

3. MINIMUM - MASS DESIGN OF A RECTANGULAR WING FOR GIVEN TORSIONAL DIVERGENCE SPEED

\subsection{Statement of the problem}

Consider a cantilever straight wing with elastic axis perpendicular to the free stream. The wing profile is assumed to be constant along the span and characterized by a lift-coefficient slope $a_{0}$. The other parameters and variables are defined in Fig. 1. The thickness $T$ of 
the skin varies along the span.

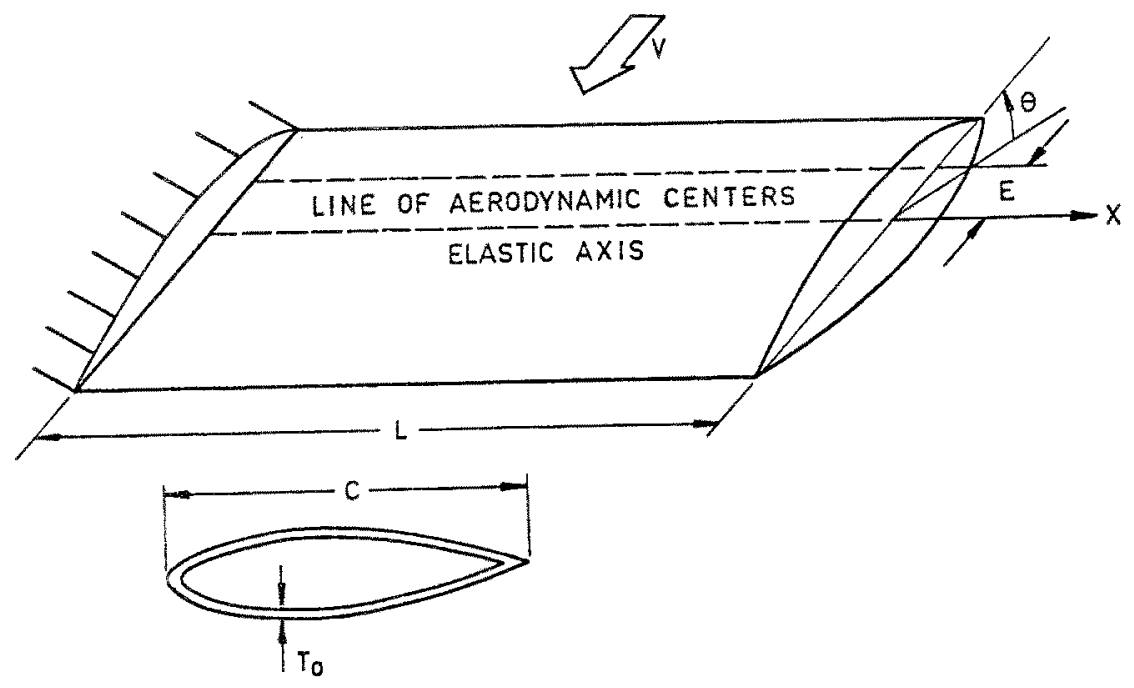

Figure 1: Unswept cantilever wing with constant chord.

The torsional divergence speed $V$ for this wing is obtained by solving the eigenvalue problem :

$$
\begin{aligned}
& \frac{d}{d X}\left(G J \frac{d \theta}{d X}\right)+\frac{1}{2} \rho V^{2} C E a_{o} \theta=0 \\
& \theta(0)=0 \\
& \left.G J \frac{d \theta}{d X}\right|_{x=L}=0
\end{aligned}
$$

$\rho$ is the free stream density. The reader is referred to Bisplinghoff, Ashley and Halfman (1955) for details. 
If we assume that the torsional stiffness of the wing is dominated by the contribution from the skin, then the torsion constant $J$ is directly proportional to the skin thickness (Bredt's formula),

$$
J=K T
$$

It is possible to rewrite eqs. (1) and (1') in a convenient, nondimensional form if we introduce that particular wing of constant skin thickness $T_{0}$ with identical cross-sectional profile and the same torsional divergence speed the so-called reference wing. We define the dimensionless quantities

$$
\mathrm{x}=\frac{\mathrm{X}}{\mathrm{L}}, \mathrm{t}=\frac{\mathrm{T}}{\mathrm{T}_{\mathrm{o}}},
$$

and denote by ()$^{\prime}$ the differentiation with respect to $x$; the eigenvalue problem (1), (1') is then rewritten as :

$$
\begin{aligned}
& \left(t \theta^{\prime}\right)^{\prime}+\omega^{2} \theta=0, \omega=\frac{\pi}{2} \\
& \theta(0)=0 \\
& \left.t \theta^{\prime}\right|_{x=1}=0
\end{aligned}
$$

The optimization problem is now stated as follows :

Minimize the functional

$$
m=\int_{0}^{2} t(x) d x
$$

representing the dimensionless skin mass, subject to the constraints (2) and (2').

Eq. (2) is split into a system of two first order differential equations after introducing a new variable $s$, as follows :

$$
\begin{aligned}
& \theta^{\prime}=\frac{s}{t} \\
& s^{\prime}=-\omega^{2} \theta
\end{aligned}
$$

with boundary conditions : 


$$
\theta(0)=0 \quad s(1)=0
$$

We introduce unknown Lagrange multipliers $\lambda_{\theta}$ and $\lambda_{S}$ and form the Hamiltonian

$$
H=t+\lambda \frac{s}{\theta}-\lambda s^{\omega^{2} \theta}
$$

Necessary conditions for an extremum of $m$ are now derived. The optimality condition is :

$$
\frac{\partial H}{\partial t} \equiv 1-\frac{\lambda_{\theta} s}{t^{2}}=0
$$

$\lambda_{\theta}, \lambda_{s}$ are solution of the system of Euler-Lagrange equations:

$$
\begin{aligned}
& \lambda_{\theta}^{\prime} \equiv-\frac{\partial H}{\partial \theta}=\omega^{2} \lambda_{s} \\
& \lambda_{s} \equiv-\frac{\partial H}{\partial s}=-\frac{\lambda_{\theta}}{t}
\end{aligned}
$$

Transversality conditions furnish two additional boundary conditions:

$$
\lambda_{\theta}(1)=0 \quad \lambda_{S}(0)=0
$$

Equations (3) and (6) together with eq. (5) and boundary conditions $\left(3^{\prime}\right)$ and $\left(6^{\prime}\right)$ form a system of 4 first order differential equations in the four unknowns $\theta, s, \lambda_{\theta}, \lambda_{s}$. This system will be solved by resorting to the so-called neighboring extremal methods; the idea is to construct a nominal solution satisfying the governing differential equations ( 3 ), the optimality condition (5) and the EulerLagrange equations (6), but not al1 the boundary conditions ( $\left.3^{\prime}\right)$ and $\left(6^{+}\right)$. 
3.2 The numerical procedure : a transition-matrix algorithm (Newton's method)

The idea is to find the relation between unspecified boundary conditions at one end and specified boundary conditions at the other end. We start by "guessing" the unknown initial conditions $s(0)$ and $\lambda_{\theta}(0)$, then integrate (3) and (6) forward from 0 to 1 , t being determined through (5). We obtain values for $s(1)$ and $\lambda_{\theta}(1)$, which in general will differ from zero. A "measure" of the variations of the final values of $s$ and $\lambda_{\theta}$ when the initial boundary conditions are perturbed is provided by the matrix $T$, the so-called transition matrix

$$
T=\left[\begin{array}{ll}
\frac{\partial s(1)}{\partial s(0)} & \frac{\partial s(1)}{\partial \lambda_{\theta}(0)} \\
\frac{\partial \lambda_{\theta}(1)}{\partial s(0)} & \frac{\partial \lambda_{\theta}(1)}{\partial \lambda_{\theta}(0)}
\end{array}\right]
$$

linking small variations of $s$ and $\lambda_{\theta}$ at 1 to small variations of the same quantities at 0

$$
\left[\begin{array}{l}
\delta s(1) \\
\delta \lambda_{\theta}(1)
\end{array}\right]=T\left[\begin{array}{l}
\delta s(0) \\
\delta \lambda_{\theta}(0)
\end{array}\right]
$$

To determine $\mathrm{T}$, we begin by computing the first variations $\delta \theta, \delta \mathrm{s}$, $\delta \lambda_{\theta}, \delta \lambda_{s}$ of the 4 quantities $\theta, s, \lambda_{\theta}, \lambda_{s}$ from the system of differential equations defining them; we obtain :

$$
\begin{aligned}
& \delta \theta^{\prime}=\frac{1}{2} \sqrt{\frac{s}{\lambda_{\theta}}}\left(\frac{\delta s}{s}-\frac{\delta \lambda_{\theta}}{\lambda_{\theta}}\right) \\
& \delta s^{\prime}=-\omega^{2} \delta \theta \\
& \delta \lambda_{\theta}^{\prime}=\omega^{2} \delta \lambda_{s} \\
& \delta \lambda_{s}^{\prime}=\frac{1}{2} \sqrt{\frac{\lambda_{\theta}}{s}}\left(\frac{\delta s}{s}-\frac{\delta \lambda_{\theta}}{\lambda_{\dot{\theta}}}\right)
\end{aligned}
$$


If $\delta s(0)$ is set equal to unity, all other perturbations being initially set to zero, integration of this system (9). together with the initial system (3), (3'), (5), (6), (6) on $[0,1]$ will yield values for $\delta s(1)$ and $\delta \lambda_{\theta}(1)$ which constitute the first column of T. Similar$1 y$, the second column of $\mathrm{T}$ is obtained by integrating both systems with, as initial conditions, $\delta \lambda_{\theta}(0)$ set equal to unity, all other perturbations equal zero, $\left(3^{\prime}\right)$ and $\left(5^{\prime}\right)$.

If the initial conditions $s(0)$ and $\lambda_{\theta}(0)$ had been correctly chosen, $s(1)$ and $\lambda_{\theta}(1)$ would both vanish. We therefore define variations of the final values $\Delta s(1)$ and $\Delta \lambda_{\theta}(1)$ which will hopefully bring the next solution closer to the desired values of $s(1)$ and $\lambda_{\theta}(1)$, defined as :

$$
\begin{aligned}
& \Delta s(1)=-\varepsilon s(1) \\
& \Delta \lambda_{\theta}(1)=-\varepsilon \lambda_{\theta}(1) \quad 0 \leq \varepsilon \leq 1
\end{aligned}
$$

Corresponding variations $\Delta s(0)$ and $\Delta \lambda_{\theta}(0)$ of initial values are obtained from (8) :

$$
\left[\begin{array}{c}
\Delta s(0) \\
\Delta \lambda_{\theta}(0)
\end{array}\right]=\mathrm{T}^{-1}\left[\begin{array}{l}
\Delta s(1) \\
\Delta \lambda_{\theta}(1)
\end{array}\right]
$$

We now take as a new guess:

$$
\begin{aligned}
& s(0)_{\text {new }}=s(0)_{01 d}+\Delta s(0) \\
& \lambda_{\theta}(0)_{\text {new }}=\lambda_{\theta}(0)_{01 d}+\Delta \lambda_{\theta}(0)
\end{aligned}
$$

and start the whole process again until $s(1)$ and $\lambda_{\theta}(1)$ have the specified value zero to the desired accuracy.

With values of $s(0)$ and $\lambda_{\theta}(0)$ initially equal to 0.3 and 6 , respectively, and $\varepsilon=1$, convergence was very smoothly obtained in 6 iterations with a relative error of $0.04:$ on the value of $t$ at the root (Fig. 2). The profile obtained is that given by the exact analytical solution (Ashley and McIntosh (1968)). 


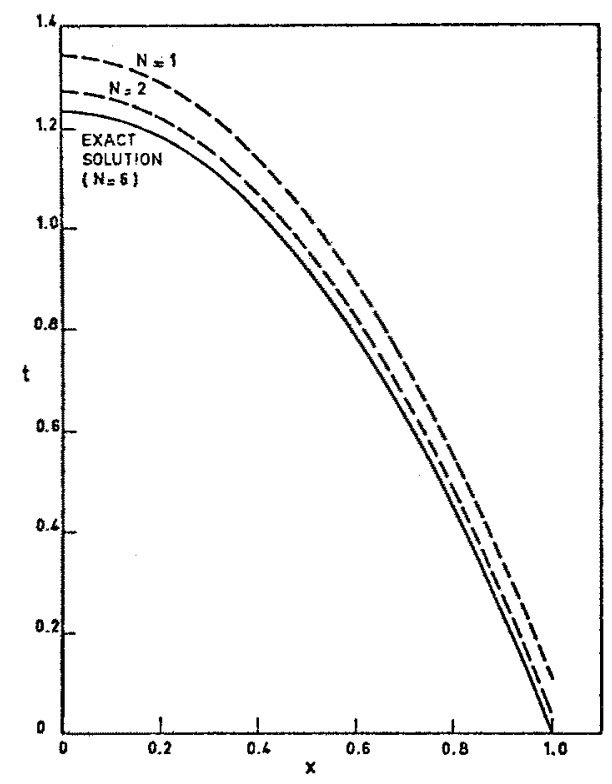

Fig. 2 : A transition-matrix procedureno minimal thickness constraint ( $\mathrm{N}=$ number of iterations $)$.

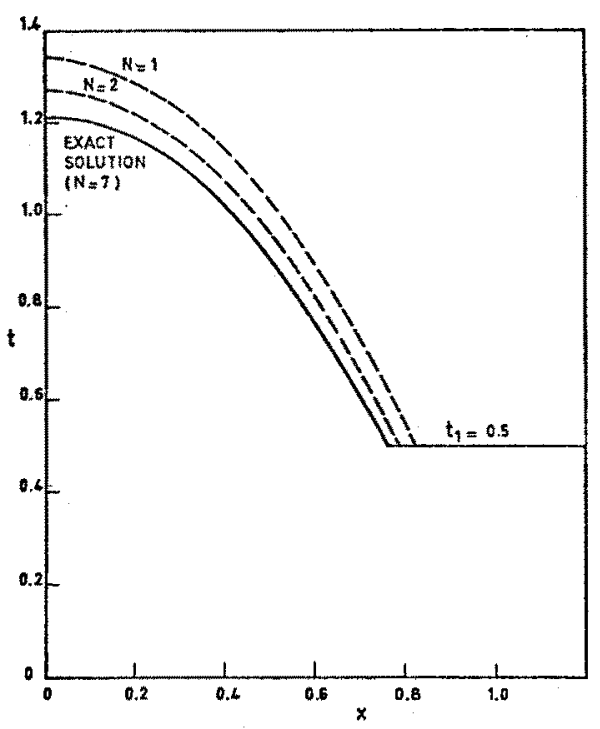

Fig. 3 : A transition-natrix procedureminimum thickness constraint

\subsection{Optimization with a minimum thickness constraint}

The more realistic case of a constraint on the value of the thickness is handled in the easiest fashion. A single statement needs to be added to the computer program, setting $t$ equal to the prescribed value $t_{\min }$ everytime its computed value $t=\sqrt{\lambda_{\theta} s}$ is smaller than $t_{\min }$. Results of the iteration procedure are shown in Fig. 3 for $t_{\min }=0.5$ where they are compared with the exact analytical solution given by Armand and Vitte (1970). The actual distribution was obtained after 7 integrations with a relative error of $0.01 \%$ on $t(0)$.

The problem just described is governed by fairly simple equations, which explains the relative ease with which the numerical solution was iteratively found. Other successful applications of this procedure can be found in the work by Armand and Vitte (1970), or Weisshaar (1970).

A slighty more complex case, for which no closed-form solution exists, is that of a vibrating simply-supported beam, first treated 
by Niordson (1965), and independently extended by Haug (1969) and Armand (1973) to handle minimum thickness constraint. The procedure described above has proven very powerful, both for the constrained and unconstrained case. Good estimates for the unknown boundary conditions were, in that case, obtained from the solution of the system of equations under consideration corresponding to the uniform reference case (therefore ignoring the optimality condition).

\section{TWO-DIMENSIONAL STRUCTURAL ELEMENTS}

The behaviour of two-dimensional structural elements such as plates and shells is described by partial differential equations in two independent variables. Optimization of such elements ot satisfy given requirements can be formulated as a problem in optimal control theory of distributedparameter systems, extension to a spatial domain $D$ of optimal control theory in which only one independent variable, the time $t$, is considered.

The domain $D$ is the planar image of the spatial structure considered, and is referred to a set of orthonormal axes $0 x$, $0 y$.

State and design variables are defined in the same fashion as before ; the deflection (in-plane or out-of-plane) and its derivatives will play the role of state variables, whereas the thickness will in some cases represent a design variable.

For a minimum-mass problem, the functional to be minimized is the surface integral of the thickness over the domain D; the constraints are in the form of partial differential equations to be satisfied inside the domain, together with equality and/or inequality constraints on functions of the state and/or design variables.

A problem of this type is a special case of a general Mayer-Bolza problem for multiple integra1s, which received authoritative treatment from Lurie (1963) in a paper which also hints at possible applications to structural optimization.

A statement of the general optimization problem as well as a derivation of the necessary conditions will be found in Armand (1972). 
Applications of the theory to structural optimization problems has been up to now very limited. The plate optimization problem, in particular, has not yet been solved in a satisfactory manner, although many researchers have considered it : reference is made to McIntosh (1974) for a comprehensive review. It shoud be mentioned at this point, however, that numerical problems are not characteristic of two-dimensional plates but also apply to axi-symmetric (one-dimensional) plates as well ; numerical difficulties encountered at the solution stage for situations involving plates, however, undoubtly correspond to problems associated with the non-existence of a global optimum.

The above observations are in accordance with the works of olhoff (1973) and Mroz (1973), who independently pointed out that the optinization problem in the case of a vibrating plate does not seem to possess a global optimum solution, but instead an infinity of local extrema.

Further research should therefore be pursued in this direction with the tools of control theory, since it is of particular interest to investigate existence and possible uniqueness of solutions to those problems for which an analytical formulation is still possible.

Numerical methods developed in the frame of optimal control of distributed-parameter systems theory and similar to methods used to solve the TPBVP's of classical optimal control have hardly been used yet and will without doubt prove extremely helpful.

A very promising approach is that described by pierson and Genalo (1974), who applied a gradient projection algorithm to obtain the optimal design of a rectangular panel subject of a flutter speed constraint. 


\section{ACKNOWLEDGEMENTS}

Research described in section 3 was performed while the author was a doctoral student at Stanford University under a National Aeronautics and Space Administration Grant.

The author also wishes to thank Professor Holt Ashley and Dr. S.C. McIntosh, Jr., whose enthusiasm for structural optimization proved contagious.

\section{REFERENCES}

Armand, J.L. and W.J. Vitte, (1970), "Foundations of Aeroelastic Optimization and Some Applications to Continuous Systems", Report SUDAAR $\mathrm{N}^{\circ} 390$, Department of Aeronautics and Astronautics, Stanford University, Stanford.

Armand, J.L., (1972), "Applications of the Theory of Optimal Control of Distributed-Parameter Systems to Structural Optimization", NASA CR2044 , June 1972.

Armand J.L., (1973), "Applications of Optimal Contro1 Theory to Structural Optimization! Analytical and Numerical Approach", Proceedings of the IUTAM Symposium on Optimization in Structural Design, Warsaw, Springer-Verlag, Berlin (to be published).

Ashley, Hi. and S.C. McIntosh, Jr., (1968), "Applications of Aeroelastic Constraints in Structural Optimization", proceedings of the 12 th International Congress of Theoretical and Applied Mechanics, Stanford, Springer-Verlag, Berlin, pp. 100-113.

Bisplinghoff, R.L., Ashley, H. and R.L. Halfman, (1955), "Aeroelasticity", Addison-Wesley Publishing Co., Reading.

Bryson, A.E., Jr. and Y.C. Ho, (1969), "Applied Optimal Control-optimization, Estimation and Control", Blaisdell Publishing Co., Watham.

Distefano, N., (1974), "Nonlinear Processes in Engineering", Academic Press, New-York.

Dixon, L.C.W., (1967), "Pontryagin's Maximum Principle Applied to the Profile of a Beam", Aeronautical Journal of the Royal Aeronautical Society, $71, \mathrm{pp} .513-515$.

Dym, C.L., (1974), "On Some Recent Approaches to Structura1 Optimization", Journa1 of Sound and Vibration, 32, pp. 49-70.

Fox, R.L., (1973), "Structural and Mechanical Design Optimization", in Optimization and Design, M. Avriel, M.J. Rijckaert and D.J. Wilde, Eds., Prentice-Hall, Englewood Cliffs, pp. 119-143.

Gallagher, R.H. and O.C. Zienkiewicz, Eds., (1973), Optimum Structural Design : Theory and Applications, John Wiley and Sons, London. 
Haug, E.J., Jr., (1969), "Optima1 Design of Structura1 Elements", Lecture Notes, Department of Mechanics and Hydraulics, The University of Iowa, Ames.

Hemp, W.S., (1973), Optimum Structures, Clarendon Press, Oxford.

Lagrange, J.L., (1770-1773), "Sur la Figure des Colonnes", Misce11anea Taurinensia, t.V., in Oeuvres de Lagrange, Tome deuxième, GauthierVillars, Paris, 1868 , pp. 125-170.

Lurie, K.A., (1963), "The Mayer-Bolza Problem for Multiple Integrals and the Optimization of the Performance of Systems with Distributed Parameters", Applied Mathematics and Mechanics (PMM), Vol. 27, $\mathrm{N}^{\circ} 5$, March 1963, pp. 1284-1299.

Majid, K.I., (1974), Optimum Design of Structures, Newnes-Butterworths, London.

McIntosh, S.C., Jr., (1974), "Structural Optimization via Optimal Control Techniques : A review", Structural Optimization Symposium AMD-VOL.7, The American Society of Mechanical Engineers, New-York, pp. 49-64

Moe, J. and K.M. Gisvold, (1971), "Optimization and Automated Design of Structures", Report SK/M 21, Division of Ship Structures, Technical University of Trondheim, Trondheim.

Mroz, S., (1973), "Multiparameter Optimal Design of Plates and Shells", Journal of Structural Mechanics, 3, pp. 371-392.

Niordson, F.I., (1965), "On the Optimal Design of a Vibration Beam", Quarterly of Applied Mathematics, 23 , pp. 45-53.

Pierson, B.L. and L.J. Genalo, (1974), "Minimum-Weight Design of a Rectangular Flat Panel Subject to a Flutter Speed Constraint", Symposium on Optimization Problems in Engineering and Economics, Naples, Dec. 1974.

Niordson, F.I. and P. Pedersen, (1972), "A Review of Optimal Structura1 Design", Proceedings of the 13 th International Congress of Theoretical and Applied Mechanics, Moscow, Springer-Verlag, Berlin, pp. 264-278.

Olhoff, N., (1973), "On Singularities, Local Optima and Formation of Stiffeners in Optimal Design of plates", proceedings of the IUTAM Symposium on Optimization in Structural Design, Warsaw, Springer-VerIag, BerIin (to be published).

Pierson, B.L., (1972), "A survey of Optimal Structural Design Under Dynamic Constraints", International Journal for Numerical Methods in Engineering, 4, pp. 49 1-499.

Prager, W. and J.E. Taylor, (1968), "Problems of Optimal Structural Design", Journal of Applied Mechanics, 35, pp. 102-106.

Schmit, L.A., Jr., (1971), "Structural Synthesis 1959-1969: A Decade of Progress", in Recent Advances in Matrix Methods of Structural Analysis and Design, R.H. Gallagher, Y. Yamada and J.T. Oden, Eds., The University of Alabama Press, Huntsvilile, pp. 565-634.

Weisshaar, T.A., (1970), "An Application of Control Theory Methods to the Optimization of Structures Having Dynamic or Aeroelastic Constraints", Report SUDAAR $\mathrm{N}^{\circ} 412$, Department of Aeronautics and Astronautics, Stanford University, Stanford. 